\title{
INVESTIGACIONES
}

\section{Predictores socio-académicos del Study Engagement en estudiantes de primer año de ingeniería*}

\author{
Socio-academic predictors of the Study Engagement \\ in engineering first year students
}

\author{
Jorge Maluenda Albornoz, Marcela Varas Contreras ${ }^{b}$, \\ Marcia Riffo-Ferradac, Alejandro Díaz-Mujica ${ }^{d}$
${ }^{a}$ Psicólogo, Magíster en Política y Gobierno. Universidad de Concepción. jorgemaluendaa@gmail.com
${ }^{b}$ Ingeniera Civil Informático. Máster en Ciencias de la Computación, Universidad de Concepción. mvaras@udec.cl.
${ }^{c}$ Psicóloga, Universidad de Concepción. marcriffo@udec.cl

${ }^{d}$ Psicólogo, Doctor en Psicología y Doctor en Ciencias de la Comunicación, Universidad de Concepción. adiazm@udec.cl

\begin{abstract}
Si bien el Study Engagement se ha vinculado con distintas variables clave para el proceso de enseñanza-aprendizaje en educación superior existe poca claridad respecto de los factores que lo afectan, especialmente, en la educación en ingeniería. El objetivo de investigación fue evaluar la capacidad predictiva de las metas académicas, el sentido de pertenencia y el apoyo de pares sobre el Study Engagement. Se realiza un diseño de tipo asociativo-predictivo de corte transversal que incorpora la medición de estas variables en 743 estudiantes de primer año de ingeniería. Los predictores más fuertes fueron las metas de aprendizaje y la pertenencia a la carrera frente a las demás metas y el apoyo. El peso de cada variable fue distinto para cada dimensión del Engagement. Con esto los resultados contribuyen a comprender diferencias en los predictores del Engagement lo que anima potenciales intervenciones diferenciadas en función de la dimensión que se requiera estimular.
\end{abstract}

Palabras claves: Compromiso académico, Metas académicas, Sentido de pertenencia, Apoyo social, Educación en ingeniería.

\section{ABSTRACT}

Although Study Engagement has been linked to different key variables for the teaching-learning process in higher education, there is a lack of clarity regarding the factors that affect it, especially in engineering education. The research objective was to evaluate the predictive capacity of academic goals, the sense of belonging and peer support on Study Engagement. A cross-sectional associative-predictive design is carried out that incorporates the measurement of these variables in 743 first-year engineering students. The strongest predictors were learning goals and career membership versus other goals and support. The weight of each variable was different for each dimension

* Este trabajo de investigación fue realizado con aportes de la beca de doctorado nacional de CONICYT: CONICYTPCHA/Doctorado Nacional/2018-21180223 y con el apoyo del proyecto FONDECYT No 1161502 "Modelo explicativo de la permanencia y el abandono de los estudios universitarios basado en procesos cognitivo-motivacionales" y "ANIDCOVID1012". Se agradece a la Faculta de Ingeniería de la Universidad de Concepción por las facilidades otorgadas para realizar el estudio. 
of the Engagement. With this, the results contribute to understanding differences in the predictors of Engagement, which encourages potential differentiated interventions depending on the dimension that needs to be stimulated.

Key words: Study engagement, Academic goals, Sense of belonging, Social support, Engineering education.

\section{INTRODUCCIÓN}

El Study Engagement ha tomado un gran realce en los últimos años debido a las relaciones encontradas entre este constructo y una variedad de factores que son relevantes para el mejoramiento en los resultados del proceso de enseñanza-aprendizaje en educación superior (Maluenda, Moraga y Díaz-Mujica, 2019).

Entendido como un meta-constructo está conformado por manifestaciones conductuales, emocionales y cognitivas de la motivación por los estudios (Fredricks, Blumenfeld y Paris, 2004; Skinner, Furrer, Marchand y Kindermann, 2008). Implica la participación y colaboración en tareas académicas, sociales y extracurriculares fundamentales para alcanzar resultados académicos positivos y prever el abandono -Engagement conductual-; respuestas positivas y negativas frente a docentes, compañeros, actividades e institución académica, como así, el vínculo que se conforme entre el estudiante y sus estudios -Engagement afectivo-; y la voluntad de realizar el esfuerzo intelectual requerido para tareas y habilidades complejas -Engagement cognitivo- (Fredricks et al., 2004; Fredricks y Mccolskey, 2012).

Distintas investigaciones han mostrado la relación del Study Engagement con variables clave del proceso de enseñanza aprendizaje como el Burnout, el rendimiento académico actual y futuro, y la satisfacción académica. En un estudio de tipo correlacional que incluyó 1661 estudiantes de distintos países europeos mostró una correlación negativa y moderada entre el Burnout y el Study Engagement (Schaufeli, Martínez, Marques, Salanova y Bakker, 2002). Otra investigación de tipo correlacional, que evaluó 277 estudiantes chilenos de primer año de medicina mostró una relación significativa entre el Study Engagement, el bienestar y el rendimiento académico (Gómez et al., 2015). En la misma línea, una investigación con 527 estudiantes españoles reportó una correlación positiva entre el Study Engagement y el rendimiento académico. Adicionalmente, mostró el rol mediador e indirecto del Study Engagement en el impacto de los obstaculizadores y facilitadores (sociales, organizacionales y personales, de cada uno) en el rendimiento futuro de los estudiantes, observándose una asociación positiva con los facilitadores y una negativa con los obstaculizadores (Salanova, Schaufeli, Martínez y Bresó, 2009).

El Study Engagement también ha mostrado relacionarse con la satisfacción académica. Una investigación con 215 estudiantes de pregrado de distintas nacionalidades mostró una correlación positiva entre el Engagement y una mayor identidad vocacional como indicador de satisfacción académica. De igual manera, un mayor Study Engagement se asoció con la toma de decisiones satisfactorias de los estudiantes (Cox, Bjornsen y Krieshok, 2015). De este modo, el Study Engagement muestra relación tanto con los resultados de aprendizaje del proceso educativo como con la buena experiencia estudiantil durante este proceso.

Por otro lado, a pesar del aumento de la investigación en esta temática, no se ha establecido con claridad cuales factores afectan el Study Engagement en estudiantes universitarios, ni cuales de estos son susceptibles de intervención.

Durante la transición desde la educación escolar a la educación superior los estudiantes deben acomodarse a una serie de cambios muy importantes. Se ven enfrentados a un 
nuevo sistema organizacional con normas explícitas e implícitas distintas, con una cultura diferente, nuevos docentes y compañeros. Además, comienzan a ajustar sus expectativas respecto de la nueva realidad de estudios aspectos que en conjunto afectan la motivación y la satisfacción académica (Figueroa y Torrado, 2013).

De este modo, factores como las metas y expectativas de los estudiantes, la interacción y el apoyo de sus pares (Cameron y Rideout, 2020; Figueroa y Torrado, 2013) pueden influir sobre el Study Engagement que exhiban.

En el contexto de la educación en ingeniería, existe menos evidencia que en el concierto educativo general, lo que añade relevancia y necesidad al estudio sobre la influencia de los distintos factores que influyen en el Study Engagement (Maluenda y Pérez, 2018; Maluenda, Flores-Oyarzo, Varas y Díaz, 2020). En esta línea, un reciente estudio cualitativo en estudiantes chilenos de ingeniería buscó explorar las razones que los estudiantes señalan como desencadenantes de altos niveles de Study Engagement. Se observaron como principales razones: Tener motivaciones asociadas al logro de objetivos $(38,1 \%)$, contar con metas claras $(11,9 \%)$ y tener claridad en las prioridades vitales $(7,1 \%)$ (Maluenda, Flores-Oyarzo, Bernardo y Díaz-Mujica, 2021).

Las metas son consideradas indicadores e importantes determinantes de la motivación de los estudiantes (Barca, Peralbo, Porto, Marcos y Brenlla, 2008). Estas dirigen el esfuerzo para conseguir los objetivos de cada estudiante y, en definitiva, por alcanzar la titulación (Tinto, 1989). Un estudiante puede estar motivado por: aprender y comprender nuevos contenidos, y así adquirir ciertas competencias (meta de aprendizaje); obtener una recompensa y reconocimiento académico (meta de logro); mantener o aumentar su autoestima, llevando a cabo tareas que si podrá realizar (metas orientadas al yo); o ser aceptado por los demás por medio de sus logros académicos (metas sociales) (García et al., 1998). Las metas académicas han sido asociadas a la contribución del sentido de eficacia personal de los estudiantes, como facilitadoras de un mejor rendimiento académico, un mayor nivel de Engagement cognitivo (Valle et al., 2009), como con la institución educativa; y una mejor integración académica como social (Saldaña y Barriga, 2010).

El apoyo social es uno de los recursos más importantes en el proceso de adaptación a la vida universitaria (Musitu, Cava y Valencia, 2003). Constituye un factor protector en este proceso ya que disminuye las consecuencias negativas, físicas y psicológicas de sucesos estresantes (Herrera, Flórez, Romero y Montalvo, 2012; Musitu, Cava y Valencia, 2003; Orcasita y Uribe, 2010), así como de experiencias perturbadoras o adversas que considera el ingreso a la enseñanza superior (Faleel, Tam, Lee, Har y Foo, 2012; Hartley, 2011; Mattanah, Ayers y Brand, 2010). El apoyo social percibido o la percepción de la persona acerca de la cantidad y calidad de apoyo social disponible en caso de que fuese necesario (Cohen y Janicki-Deverts, 2010; Orcasita y Uribe, 2010), juega un papel importante en favorecer la integración del estudiante tanto al contexto social como académico, pudiendo afectar el Engagement del estudiante con sus estudios.

El sentido de pertenencia se refiere al grado con que un individuo liga su sentido del yo con el de un grupo, piensa en sí mismo y en este grupo en términos similares y se define a sí mismo como un miembro de este (Tyler y Blander, 2000). De esta forma, comparte los valores y metas del grupo, siente deseos de permanecer en este y se percibe aceptado, valorado, perteneciente y parte de la vida como de las actividades que se desarrollan (Goodenow, 1993). Cuando en el contexto educativo existe una conexión del estudiante con el entorno de aprendizaje académico formal y con el entorno social como cultural de 
esta comunidad, el sentido de pertenencia puede convertirse en un deseo de quedarse y completar sus objetivos educativos (Torres y Solberg, 2001). En educación escolar se ha observado que el sentido de pertenencia es un buen predictor del Study Engagement, la valoración de las tareas académicas, expectativas de éxito e interés general por el centro de estudios. Asimismo, se ha demostrado una relación positiva con la preparación, asistencia y participación en clases como una conducta apropiada en el aula, todos indicadores del Study Engagement (Goodenow, 1993; Finn, 1993). Bajo esta misma línea, pero en el contexto universitario, el sentido de pertenencia a la carrera involucra sentirse un miembro relevante y respetado en su programa de estudios (Alkan, 2014). Surge en base al juicio que realiza el estudiante del nuevo escenario universitario y su ajuste, como también, del sentido de afiliación e identificación con la comunidad universitaria (Hoffman, Richmond, Morrow y Salomone, 2003). En investigaciones previas de tipo asociativo-predictivo en estudiante universitarios, se ha observado que la identificación organizacional -como indicador de pertenencia- es un predictor fuerte para el Study Engagement (Di Battista, Pivetti y Berti, 2014; Wilkins, Mohsin, Kratochvil y Stephens, 2016). Además, este tiene un efecto sobre el compromiso institucional que se expresa en el deseo de participar en actividades y en manifestar su identidad vistiendo logos e insignias, además de trabajar más duro por las metas colectivas e involucrarse más en conductas altruistas y de ayuda con sus pares (Boros y Curseu, 2012).

Basada en lo anteriormente planteado, la presente investigación pretende evaluar la capacidad predictiva de las metas académicas, el sentido de pertenencia en la carrera y el apoyo percibido de pares sobre el Study Engagement con la carrera en estudiantes de ingeniería de reciente ingreso. Con ello, se espera contribuir al esclarecimiento sobre aquellas variables que contribuyen a afectar positivamente el Study Engagement, pero, sobre todo, aquellas que pueden ser modificables mediante intervención como son las metas académicas, el apoyo de los pares y el sentirse perteneciente en la carrera.

\section{MÉTODO}

\subsection{PARTICIPANTES}

Se realizó un muestreo incidental que incorporó a los estudiantes de ingeniería de primer año, de reciente ingreso, disponibles en la jornada de inducción a la universidad realizada la primera semana de clases por la Unidad de Educación en Ingeniería. Los participantes fueron 743 estudiantes de ingeniería, equivalentes a un 67,6\% del total de la cohorte. Un $74,6 \%$ son estudiantes hombres y un $25,4 \%$ mujeres, cuya media de edad fue 18,1 años y su desviación típica 0,8 años.

\subsection{DISEÑO Y PROCEDIMIENTOS}

El estudio se realizó con un diseño de tipo asociativo- predictivo, realizado en un corte transversal de tiempo (Ato, López y Benavente, 2013). Se realizó la evaluación de las relaciones entre el sentido de pertenencia en la carrera, el apoyo de pares, las metas académicas y el Study Engagement con la carrera, para luego evaluar la capacidad predictiva de las primeras tres variables sobre el Study Engagement. 


\subsection{RECOLECCIÓN DE DATOS}

Las aplicaciones fueron realizadas en las salas donde los estudiantes realizan regularmente las actividades de inducción a la vida universitaria, una semana antes del inicio de clases. Esta se efectuó a través de cuestionarios físicos aplicados simultáneamente por los psicólogos de la Unidad de Educación en Ingeniería, a cargo de los procesos de inducción de los estudiantes de primer año, previa capacitación en su administración y uso. Antes de la aplicación se realizó la firma del consentimiento informado que contenía los detalles sobre los fines del estudio, el carácter voluntario del estudio, la posibilidad de retirarse sin explicación alguna cuando fuese deseado por el participante, la no divulgación de información personal ni de identificación, la publicación de resultados agregados y la no existencia de efectos perniciosos de esta investigación sobre su salud. No se entregó incentivo alguno por participar. La información fue recolectada la primera semana de marzo del año 2019 (primer semestre académico en Chile).

\subsection{INSTRUMENTOS}

\subsubsection{Study Engagement}

La medición del Study Engagement en la carrera será realizada a partir del University Student Engagement Inventory. Este instrumento mide el Study Engagement de los estudiantes a partir de las 3 dimensiones (cognitiva, emocional y conductual) propuestas por Fredricks et al., (2004). Ha sido desarrollado para evaluar el Study Engagement en el contexto universitario, teniendo su estudio original de validación en Portugal en idioma Inglés (Maroco, Maroco, Campos y Fredricks, 2016). El instrumento cuenta con una versión adaptada lingüística y culturalmente, y cuyas propiedades psicométricas fueron evaluadas en estudiantes universitarios de ingeniería alcanzando adecuados índices de validez de constructo, de criterio y confiabilidad (Maluenda, Varas, Díaz y Bernardo, 2020). Se basa en el autoreporte del estudiante y cuenta con 15 ítems distribuidos en 3 factores: a) Involucramiento en actividades académicas (dimensión conductual, 5 ítems), b) Afectos frente a la carrera (dimensión emocional, 5 ítems) y c) Esfuerzo cognitivo en tareas desafiantes (dimensión cognitiva, 5 ítems), cuya respuesta se realiza en escala Likert con valores que fluctúan entre 1 (Nunca) y 5 (Siempre). El instrumento adaptado a estudiantes de ingeniería chilenos mostró una adecuada correspondencia factorial y un ajuste del modelo ( $\mathrm{CFI}=.903$; TLI=.883; RMSEA=.056), además de reflejar validez de criterio. El índice de fiabilidad (alfa de Cronbach) del instrumento universitario reportó un $\alpha=.779$ (Maluenda, Varas, Díaz y Bernardo, 2020).

\subsubsection{Metas académicas}

Las metas académicas fueron evaluadas a partir del Cuestionario de Evaluación de Metas Académicas (CEMA). Este ha sido probado en población universitaria mexicana por Gaeta, Cavazos, Sánchez y Rosário, (2015). El cuestionario cuenta con 42 ítems, cada uno de los cuales es una afirmación sobre los motivos que un estudiante tiene para realizar una determinada acción. En cada ítem el estudiante debe señalar su nivel de acuerdo mediante la señalización de una de las siguientes cinco opciones: "Nunca - Casi Nunca - Algunas veces 
- Casi siempre - Siempre" permitiendo diferenciar los tipos de metas de los estudiantes. Los ítems se distribuyen en cuatro dimensiones: a) Metas orientadas al aprendizaje, b) Metas orientadas al yo, c) Metadas orientadas a la valoración social y d) Metas de Logro y Recompensa. La escala mostró una adecuada correspondencia factorial y un ajuste del modelo (CFI=.94; BBNNFI=.94; RMSEA=.05), además de cumplir los criterios de validez convergente y discriminante. Los índices de fiabilidad (alfa de Cronbach) del instrumento universitario oscilan desde $\alpha=.78$ hasta $\alpha=.89$ (Gaeta, Cavazos, Sánchez y Rosário, 2015).

\subsubsection{Sentido de pertenencia en la carrera y apoyo de pares}

En el caso de estas variables, se utilizaron ítems desarrollados por los investigadores. Para el sentido de pertenencia en la carrera se utilizaron 3 reactivos: "Me siento incluido en la carrera", "Me siento parte de la carrera", "Me siento incluido en ingeniería". Se realizó un análisis factorial exploratorio con rotación oblimin del conjunto de ítems obteniendo un solo factor. Las cargas factoriales fluctuaron entre .842 y .964. El análisis de la confiabilidad mediante el índice $\alpha$ de Cronbach mostró un valor de .920 mientras que la $\omega$ de McDonald mostró un valor de .922. Para el apoyo percibido de pares se utilizaron 2 ítems: "Tengo relación con algún compañero que seguramente me ayudaría en el curso de mi carrera, "Tengo relación con algún grupo de compañeros que seguramente me ayudaría en el curso de mi carrera”. Se realizó un análisis factorial exploratorio con rotación oblimin del conjunto de ítems obteniendo un solo factor, al cual cada ítem contribuye con una carga factorial de .600. El análisis de la confiabilidad mediante el índice $\alpha$ de Cronbach mostró un valor de .626 mientras que la $\omega$ de McDonald mostró un valor de .630. En ambos casos, el modo de respuesta fue en escala Likert con 1 (Nunca) y 5 (Siempre). Todos los análisis fueron realizados con el software JASP 0.86 .

\subsection{ANÁLISIS DE DATOS}

Se realizó un examen preliminar de los datos con la finalidad de verificar el cumplimiento de los supuestos asociados a la regresión lineal múltiple, los cuales, estuvieron dentro de los márgenes aceptados en la literatura. Luego, se examinaron las correlaciones bivariadas a través del coeficiente de correlación de Pearson con la finalidad de conocer el grado de asociación entre las variables y verificar su potencial de ingreso en un modelo de regresión lineal. Finalmente, se utilizó la regresión lineal múltiple con método stepwise para evaluar la capacidad predictiva de las variables independientes sobre la variable dependiente, y obtener distintos modelos en función de la proporción de varianza explicada por las variables en su conjunto. Los análisis anteriormente indicados fueron realizados a partir del software SPSS versión 22.

\section{RESULTADOS}

\subsection{CORRELACIONES}

Antes de la realización de los análisis, se examinó el cumplimiento de los supuestos necesarios para la regresión lineal, dando cumplimiento a cada uno de ellos. A continuación, 
se analizaron las correlaciones entre las distintas variables incorporadas. El análisis a partir de correlaciones bivariadas (Pearson) entre las variables consideradas mostró relaciones fuertes entre Study Engagement global, sentido de pertenencia en la carrera y metas de aprendizaje. Por su parte, las metas sociales mostraron una relación fuerte respecto de las metas orientadas al yo y las metas de logro. El apoyo de pares fue la única variable que no mostró relaciones con las metas de aprendizaje, sociales y orientadas al yo. Las únicas relaciones negativas encontradas fueron entre las metas de logro y el apoyo de pares, y entre las metas orientadas al yo respecto del Engagement con la carrera, el sentido de pertenencia en la carrera y las metas de aprendizaje (Tabla 1).

Tabla 1. Correlaciones entre las variables incluidas

\begin{tabular}{|c|c|c|c|c|c|c|c|}
\hline & $\begin{array}{l}\text { Engagement } \\
\text { carrera }\end{array}$ & $\begin{array}{l}\text { Sentido de } \\
\text { pertenencia }\end{array}$ & $\begin{array}{c}\text { M. } \\
\text { Aprendizaje }\end{array}$ & $\begin{array}{c}\text { M. } \\
\text { Social }\end{array}$ & $\begin{array}{c}\text { M. Orientadas } \\
\text { al yo }\end{array}$ & $\begin{array}{c}\text { M. } \\
\text { Logro }\end{array}$ & $\begin{array}{l}\text { Apoyo } \\
\text { pares }\end{array}$ \\
\hline $\begin{array}{l}\text { Engagement } \\
\text { carrera }\end{array}$ & & $.420 * *$ & $.598 * *$ & $.078^{*}$ & $-.152 * *$ & $.161 * *$ & $.150 * *$ \\
\hline $\begin{array}{l}\text { Sentido de } \\
\text { pertenencia }\end{array}$ & $.420 * *$ & & $.292 * *$ & $.111^{* *}$ & -.058 & $.153^{* *}$ & $.181^{* *}$ \\
\hline $\begin{array}{c}\text { M. } \\
\text { Aprendizaje }\end{array}$ & $.598 * *$ & $.292 * *$ & & $.111^{* *}$ & $-.018 * *$ & $.181 * *$ & .033 \\
\hline M. Social & $.078 *$ & $.111^{* *}$ & $.111^{* *}$ & & $.536 * *$ & $.381^{* *}$ & .068 \\
\hline $\begin{array}{c}\text { M. Orientada } \\
\text { al yo }\end{array}$ & $-.152 * *$ & -.058 & $-.108 * *$ & $.536 * *$ & & $.170^{* *}$ & .011 \\
\hline M. Logro & $.161 * *$ & $.153 * *$ & $.181 * *$ & $.381 * *$ & $.170 * *$ & & $-.102 * *$ \\
\hline $\begin{array}{l}\text { Apoyo de } \\
\text { pares }\end{array}$ & $.150 * *$ & $.181 * *$ & .033 & .068 & .011 & $-.102 * *$ & \\
\hline
\end{tabular}

*Correlación significativa al .05 (2 colas).

** Correlación significativa al .01 (2 colas).

Fuente. Elaboración propia.

\subsection{ENGAGEMENT GLOBAL}

En primer lugar, se realizó una regresión múltiple con método stepwise en la que se introdujeron como variables predictoras las metas (aprendizaje, social, logro y orientadas al yo), el sentido de pertenencia en la carrera y el apoyo percibido de pares, y donde la variable dependiente fue el Study Engagement global con la carrera. La regresión reportó 4 modelos posibles (Tabla 2) que explicaron entre un 35,8 y un $43,8 \%$ de la varianza total. 
Estudios Pedagógicos XLVII N $1:$ 235-250, 2021

PREDICTORES SOCIO-ACADÉMICOS DEL STUDY ENGAGEMENT EN ESTUDIANTES DE PRIMER AÑO DE INGENIERÍA

Tabla 2. Engagement global: Regresión múltiple, modelos y porcentajes de varianza

\begin{tabular}{|c|c|c|c|}
\hline Modelo & $\mathrm{r}^{2}$ & Error estándar de la estimación & Cambio en $\mathrm{r}^{2}$ \\
\hline 1 & .358 & 4.92916 & .358 \\
\hline 2 & .424 & 4.67201 & .066 \\
\hline 3 & .431 & 4.64535 & .007 \\
\hline 4 & .438 & 4.62076 & .007 \\
\hline
\end{tabular}

Fuente. Elaboración propia.

Las variables que mostraron explicar mejor el Study Engagement global fueron las metas de aprendizaje $(\beta=.520, p<.01)$ y el sentido de pertenencia en la carrera $(\beta=.269$, $\mathrm{p}<.01$ ) que, en conjunto, explicaron un $42,2 \%$ de la varianza (modelo 2, tabla 3). Los modelos que incorporaron variables adicionales mostraron un aumento en la varianza explicada adicional marginal.

Tabla 3. Engagement global: Significación y tamaño de las relaciones por modelo

\begin{tabular}{|c|c|c|c|c|c|c|}
\hline & Modelo & B & Error estándar & Beta & $\mathrm{t}$ & Sig. \\
\hline 1 & $\begin{array}{l}\text { Constante } \\
\text { M. Aprendizaje }\end{array}$ & $\begin{array}{c}31.826 \\
2.828\end{array}$ & $\begin{array}{c}1.180 \\
.136\end{array}$ & .598 & $\begin{array}{l}26.981 \\
20.754\end{array}$ & $\begin{array}{l}\mathrm{P}<.001 \\
\mathrm{P}<.001\end{array}$ \\
\hline 2 & $\begin{array}{l}\text { Constante } \\
\text { M. Aprendizaje } \\
\text { Sent. Pertenencia }\end{array}$ & $\begin{array}{c}25.952 \\
2.457 \\
2.155\end{array}$ & $\begin{array}{l}1.281 \\
.135 \\
.229\end{array}$ & $\begin{array}{l}.520 \\
.269\end{array}$ & $\begin{array}{c}20.263 \\
18.201 \\
9.404\end{array}$ & $\begin{array}{l}\mathrm{P}<.001 \\
\mathrm{P}<.001 \\
\mathrm{P}<.001\end{array}$ \\
\hline 3 & $\begin{array}{l}\text { Constante } \\
\text { M. Aprendizaje } \\
\text { Sent. Pertenencia } \\
\text { Apoyo pares }\end{array}$ & $\begin{array}{c}25.208 \\
2.466 \\
2.025 \\
.368\end{array}$ & $\begin{array}{l}1.295 \\
.134 \\
.232 \\
.117\end{array}$ & $\begin{array}{l}.522 \\
.252 \\
.087\end{array}$ & $\begin{array}{c}19.463 \\
18.369 \\
8.740 \\
3.144\end{array}$ & $\begin{array}{l}\mathrm{P}<.001 \\
\mathrm{P}<.001 \\
\mathrm{P}<.001 \\
\mathrm{P}=.002\end{array}$ \\
\hline 4 & $\begin{array}{l}\text { Constante } \\
\text { M. Aprendizaje } \\
\text { Sent. Pertenencia } \\
\text { Apoyo pares } \\
\text { M. Orientadas al yo }\end{array}$ & $\begin{array}{c}27.169 \\
2.428 \\
2.003 \\
.375 \\
-.217\end{array}$ & $\begin{array}{l}1.441 \\
.134 \\
.231 \\
.116 \\
.071\end{array}$ & $\begin{array}{l}.514 \\
.250 \\
.089 \\
-.083\end{array}$ & $\begin{array}{c}18.854 \\
18.095 \\
8.688 \\
3.220 \\
-3.037\end{array}$ & $\begin{array}{l}\mathrm{P}<.001 \\
\mathrm{P}<.001 \\
\mathrm{P}<.001 \\
\mathrm{P}<.001 \\
\mathrm{P}=.002\end{array}$ \\
\hline
\end{tabular}

Fuente. Elaboración propia. 


\subsection{ENGAGEMENT: DIMENSIÓN CONDUCTUAL}

A continuación, se realizó una regresión múltiple con método stepwise en la que se introdujeron como variables predictoras las metas (aprendizaje, social, logro y orientadas al yo), el sentido de pertenencia en la carrera y el apoyo percibido de pares, y donde la variable dependiente, esta vez, fue la dimensión conductual del Study Engagement con la carrera. La regresión reportó 6 modelos posibles (Tabla 4) que explicaron entre un 31,5 y un $36,4 \%$ de la varianza total.

Tabla 4. Engagement conductual: Regresión múltiple, modelos y porcentajes de varianza

\begin{tabular}{|c|c|c|c|}
\hline Modelo & $\mathrm{r}^{2}$ & Error estándar de la estimación & Cambio en $\mathrm{r}^{2}$ \\
\hline 1 & .315 & 1.91105 & .315 \\
\hline 2 & .330 & 1.89123 & .015 \\
\hline 3 & .340 & 1.87847 & .010 \\
\hline 4 & .350 & 1.86557 & .010 \\
\hline 5 & .357 & 1.85528 & .008 \\
\hline 6 & .364 & 1.84654 & .007 \\
\hline
\end{tabular}

Fuente. Elaboración propia.

La variable que mostró explicar mejor el Study Engagement conductual fue la meta de aprendizaje $(\beta=.561, \mathrm{p}<.01)$ explicando un $31,5 \%$ de la varianza por si sola (modelo 1$)$. Los modelos que incorporaron variables adicionales mostraron un aumento en la varianza explicada adicional marginal.

Tabla 5. Engagement conductual: Significación y tamaño de las relaciones por modelo

\begin{tabular}{|c|l|c|c|c|c|c|}
\hline \multicolumn{2}{|c|}{ Modelo } & B & Error estándar & Beta & t & Sig. \\
\hline \multirow{2}{*}{1} & Constante & 12.184 & .457 & & 26.640 & $\mathrm{P}<.001$ \\
& M. Aprendizaje & .995 & .053 & .561 & 18.843 & $\mathrm{P}<.001$ \\
\hline \multirow{3}{*}{2} & Constante & 11.132 & .518 & & 21.463 & $\mathrm{P}<.001$ \\
& M. Aprendizaje & .929 & .055 & .524 & 17.000 & $\mathrm{P}<.001$ \\
& Sent. Pertenencia & .385 & .093 & .128 & 4.158 & $\mathrm{P}<.001$ \\
\hline \multirow{4}{*}{3} & Constante & 10.198 & .584 & & 17.469 & $\mathrm{P}<.001$ \\
& M. Aprendizaje & .902 & .055 & .509 & 16.449 & $\mathrm{P}<.001$ \\
& Sent. Pertenencia & .352 & .093 & .117 & 3.800 & $\mathrm{P}<.001$ \\
& M. Logro & .151 & .045 & .102 & 3.395 & $\mathrm{P}=.001$ \\
\hline
\end{tabular}




\begin{tabular}{|l|l|c|c|c|c|c|}
\hline \multirow{4}{*}{4} & Constante & 10.926 & .018 & & 17.691 & $\mathrm{P}<.001$ \\
M. Aprendizaje & .879 & .055 & .495 & 16.007 & $\mathrm{P}<.001$ \\
Sent. Pertenencia & .336 & .092 & .112 & 3.650 & $\mathrm{P}<.001$ \\
M. Logro & .182 & .045 & .122 & 4.027 & $\mathrm{P}<.001$ \\
M. Orientadas al yo & -.100 & .029 & -.102 & -3.420 & $\mathrm{P}=.001$ \\
\hline & & & & 18.050 & $\mathrm{P}<.001$ \\
Constante & 11.308 & .626 & .484 & 15.627 & $\mathrm{P}<.001$ \\
M. Aprendizaje & .859 & .055 & .104 & 3.391 & $\mathrm{P}<.001$ \\
Sent. Pertenencia & .312 & .092 & .091 & 2.883 & $\mathrm{P}=.004$ \\
M. Logro & .136 & .047 & -.160 & -4.559 & $\mathrm{P}<.001$ \\
M. Orientadas al yo & -.158 & .035 & .115 & 3.093 & $\mathrm{P}=.002$ \\
\hline M. Sociales & .113 & .037 & & 17.663 & $\mathrm{P}<.001$ \\
\hline & & & & \\
Constante & 11.092 & .628 & .487 & 15.792 & $\mathrm{P}<.001$ \\
M. Aprendizaje & .865 & .055 & .089 & 2.874 & $\mathrm{P}=.004$ \\
Sent. Pertenencia & .267 & .093 & .085 & 2.700 & $\mathrm{P}=.007$ \\
M. Logro & .127 & .047 &. .159 & -4.551 & $\mathrm{P}<.001$ \\
M. Orientadas al yo & -.157 & .034 & .112 & 3.034 & $\mathrm{P}=.002$ \\
M. Sociales & .111 & .036 & .085 & 2.890 & $\mathrm{P}=.004$ \\
\hline Apoyo Pares & .135 & .047 & & & \\
\hline
\end{tabular}

Fuente. Elaboración propia.

\subsection{ENGAGEMENT: DIMENSIÓN AFECTIVA}

Se realizó una regresión múltiple con método stepwise en la que se introdujeron como variables predictoras las metas (aprendizaje, social, logro y orientadas al yo), el sentido de pertenencia en la carrera y el apoyo percibido de pares, y donde la variable dependiente, esta vez, fue la dimensión afectiva del Study Engagement con la carrera. La regresión reportó 2 modelos posibles (Tabla 6) que explicaron entre un 17,9 y un $25,2 \%$ de la varianza total.

Tabla 6. Engagement afectivo: Regresión múltiple, modelos y porcentajes de varianza

\begin{tabular}{|c|c|c|c|}
\hline Modelo & $\mathrm{r}^{2}$ & Error estándar de la estimación & Cambio en $\mathrm{r}^{2}$ \\
\hline 1 & .179 & 2.51376 & .179 \\
\hline 2 & .252 & 2.40091 & .073 \\
\hline
\end{tabular}

Fuente. Elaboración propia.

Las variables que mostraron explicar mejor el Study Engagement afectivo fueron el sentido de pertenencia en la carrera $(\beta=.340, \mathrm{p}<.01)$ y las metas de aprendizaje $(\beta=.283$, $\mathrm{p}<.01$ ) explicando un $25,2 \%$ de la varianza (modelo 2 ). Las demás variables no fueron estadísticamente significativas en la explicación de la variable dependiente. 
Tabla 7. Engagement afectivo: Significación y tamaño de las relaciones por modelo

\begin{tabular}{|c|l|c|c|c|c|c|}
\hline \multicolumn{2}{|c|}{ Modelo } & B & Error estándar & Beta & t & Sig. \\
\hline \multirow{2}{*}{1} & Constante & 9.228 & .503 & & & $\mathrm{P}<.001$ \\
& Sent. Pertenencia & 1.529 & .118 & .423 & 18.342 & $\mathrm{P}<.001$ \\
\hline \multirow{2}{*}{2} & Constante & 5.323 & .658 & & 8.088 & $\mathrm{P}<.001$ \\
& Sent. Pertenencia & 1.231 & .118 & .340 & 10.448 & $\mathrm{P}<.001$ \\
& M. Aprendizaje & .602 & .069 & .283 & 8.682 & $\mathrm{P}<.001$ \\
\hline
\end{tabular}

Fuente. Elaboración propia.

\subsection{ENGAGEMENT: DIMENSIÓN COGNITIVA}

Se realizó una regresión múltiple con método stepwise en la que se introdujeron como variables predictoras las metas (aprendizaje, social, logro y orientadas al yo), el sentido de pertenencia en la carrera y el apoyo percibido de pares, y donde la variable dependiente fue la dimensión cognitiva del Study Engagement con la carrera. La regresión reportó 3 modelos posibles (Tabla 8) que explicaron entre un 18,1 y un $20,1 \%$ de la varianza total.

Tabla 8. Engagement cognitivo: Regresión múltiple, modelos y porcentajes de varianza

\begin{tabular}{|c|c|c|c|}
\hline Modelo & $\mathrm{r}^{2}$ & Error estándar de la estimación & ${\text { Cambio en } \mathrm{r}^{2}}^{2}$ \\
\hline 1 & .181 & 2.82102 & .181 \\
\hline 2 & .197 & 2.79501 & .016 \\
\hline 3 & .201 & 2.88913 & .004 \\
\hline
\end{tabular}

Fuente. Elaboración propia.

La variable que mostró explicar mejor el Study Engagement cognitivo fue la meta de aprendizaje ( $\beta=.425, \mathrm{p}<.01$ ), explicando un $18,1 \%$ de la varianza (modelo 1 ). Los modelos que incorporaron variables adicionales mostraron un aumento en la varianza explicada adicional marginal. 
Tabla 9. Engagement cognitivo: Significación y tamaño de las relaciones por modelo

\begin{tabular}{|l|l|c|c|c|c|c|}
\hline \multicolumn{2}{|c|}{ Modelo } & B & Error estándar & Beta & t & Sig. \\
\hline \multirow{2}{*}{1} & Constante & 10.965 & .675 & & 16.243 & $\mathrm{P}<.001$ \\
& M. Aprendizaje & 1.019 & .078 & .425 & 13.062 & $\mathrm{P}<.001$ \\
\hline \multirow{2}{*}{2} & Constante & 9.496 & .766 & & 12.394 & $\mathrm{P}<.001$ \\
& M. Aprendizaje & .926 & .081 & .387 & 11.464 & $\mathrm{P}<.001$ \\
& Sent. Pertenencia & .539 & .137 & .133 & 3.932 & $\mathrm{P}<.001$ \\
\hline \multirow{3}{*}{3} & Constante & 10.339 & .867 & & 11.927 & $\mathrm{P}<.001$ \\
& M. Aprendizaje & .950 & .081 & .397 & 11.666 & $\mathrm{P}<.001$ \\
& Sent. Pertenencia & .569 & .138 & .140 & 4.138 & $\mathrm{P}<.001$ \\
& M. Logro & -.136 & .066 & -.068 & -2.063 & $\mathrm{P}=0.39$ \\
\hline
\end{tabular}

Fuente. Elaboración propia.

\section{CONCLUSIONES Y DISCUSIÓN}

Los resultados del presente trabajo de investigación permitieron lograr el objetivo planteado inicialmente. Se evaluó la capacidad predictiva de las metas académicas, el sentido de pertenencia en la carrera y el apoyo percibido de pares sobre el Study Engagement con la carrera en estudiantes de ingeniería de reciente ingreso.

En primer lugar, las relaciones más fuertes se observaron entre las metas de aprendizaje, el sentido de pertenencia en la carrera y el Study Engagement. Es más, tanto a nivel de Engagement global como por dimensión, el sentido de pertenencia y las metas de aprendizaje fueron los predictores más importantes donde su contribución en la explicación de varianza fue sustantivamente superior respecto de los demás predictores significativos. Estos resultados reflejan que, cuando un estudiante es motivado por el aprendizaje en sí mismo y se siente perteneciente en la carrera, manifiesta un Study Engagement más elevado. Los resultados relacionados con el sentido de pertenencia en la carrera son coherentes con investigación previa que ha reportado un vínculo entre la pertenencia a la institución educativa y el Study Engagement de los estudiantes (Itzhaki, 2018).

Por otro lado, la única variable que mostró ser un predictor negativo del Study Engagement fue la meta orientada al yo. Sin embargo, su aporte a la explicación de la varianza total del Study Engagement fue pequeño, y solo ocurre en el caso del Study Engagement global $(0,07 \%)$ y del Study Engagement conductual (1\%).

Si bien los demás tipos de metas académicas y el apoyo de los pares tuvieron relación significativa con el Study Engagement, estas variables no mostraron un aporte sustantivo en la explicación del fenómeno. Vinculado a lo anterior, es importante indicar que se observaron relaciones fuertes entre las metas sociales respecto de las metas orientadas al yo y metas de logro. Esto podría indicar que las metas académicas vinculadas a una motivación con control externo (aprobación social, rendir, lograr) no contribuyen al Study Engagement. En la misma línea, en investigaciones previas, se ha observado que el favorecimiento de la motivación autónoma favorecen el Study Engagement (Díaz-mujica et al., 2018; Pérez, Cobo, Matos, De Valle y Díaz, 2017). 
Por otro lado, la percepción de apoyo por parte de los pares, si bien arrojó una correlación pequeña respecto del Study Engagement, tampoco mostró predecirlo de manera sustantiva. Este efecto, contrario a evidencia previa (Itzhaki, 2018; Wang y Eccles, 2012), puede deberse a un factor gravitante que se constituye en una particularidad del presente estudio: los estudiantes que participaron son aquellos de reciente ingreso, que no se conocen ni han establecido relaciones de mayor profundidad con otros, y cuya experiencia universitaria es aún muy restringida. Por lo tanto, podría pensarse que, el apoyo social de pares no juega un papel sustancial respecto del Study Engagement al inicio de la universidad en estudiantes de ingeniería, aspecto que podría cambiar en etapas más avanzadas.

Otro resultado que destaca son las diferencias en la importancia relativa de cada conjunto de variables frente al Study Engagement por dimensiones. Tanto en el Engagement global, como en el conductual y cognitivo, las metas de aprendizaje son el predictor más relevante del conjunto de predictores significativos. Al respecto, es posible interpretar que, la orientación de los estudiantes por aprender (meta de aprendizaje) estaría vinculada con el Study Engagement conductual que, desde la operacionalización mantenida en esta investigación, significa una percepción de mayor participación e involucramiento en las actividades académicas (Maroco et al., 2016; Maluenda, Varas, Díaz y Bernardo (2020). De manera similar, la orientación de los estudiantes por aprender estaría vinculada con el Engagement cognitivo, es decir, la percepción de esfuerzo cognitivo involucrado en abordar las tareas complejas y/o de mayor dificultad (Maroco et al., 2016; Maluenda et al., 2020).

La diferencia se observa solo en el caso del Engagement afectivo, donde si bien las metas de aprendizaje y el sentido de pertenencia a la carrera aportan significativa y sustantivamente a la explicación del Engagement, el sentido de pertenencia en la carrera tiene un mayor peso que las metas de aprendizaje. La explicación frente a este resultado puede estar relacionada con la concordancia entre el sentirse perteneciente y una afectividad positiva frente a la carrera. Esto debido a que, en la presente investigación, el Engagement afectivo ha sido definido como las reacciones afectivas positivas frente a la carrera (Maroco et al., 2016; Maluenda et al., 2020). Este resultado puede ser particularmente interesante, si se considera que otras investigaciones han mostrado que, en estudiantes de ingeniería de primer año, el Engagement afectivo es el predictor más fuerte de las intenciones de abandonar los estudios (Maluenda, López, Varas, Bernardo, Díaz y Moraga, 2019).

A partir de los resultados ya descritos es posible señalar que, la presente investigación contribuye a esclarecer el aporte de estas distintas variables sobre el Study Engagement en un contexto especifico (primer año de ingeniería) donde el interés ha crecido dada su vinculación con el favorecimiento del desempeño y la disminución del burnout académico y el abandono de los estudios. El desconocimiento sobre factores que puedan contribuir aumentar el Engagement realza el aporte de la presente investigación, que entrega evidencias sobre variables susceptibles de modificación/intervención que podrían favorecerlo. Adicionalmente, el ejercicio de desagregación del Engagement en sus dimensiones permite la detección de diferencias en el aporte de cada variable en la comprensión del fenómeno.

Por otro lado, se observa como una limitación de esta investigación el uso de una reducida cantidad de ítems para evaluar el apoyo percibido de pares (dos ítems) lo que podría afectar las propiedades psicométricas de esta medida. Ello implica la necesidad de evaluar el apoyo social con a través de un instrumento con mejores cualidades para una futura investigación, que permitan acceder a conclusiones con mayor grado de rigurosidad y certeza. 
Estudios Pedagógicos XLVII N 1: 235-250, 2021

PREDICTORES SOCIO-ACADÉMICOS DEL STUDY ENGAGEMENT EN ESTUDIANTES DE PRIMER AÑO DE INGENIERÍA

\section{REFERENCIAS BIBLIOGRÁFICAS}

Alkan, N. (2014). Humor, loneliness and acceptance: Predictors of university drop-out intentions. Precedia Social and Behavioral Sciences, 152, 1079-1086. https://doi.org/10.1016/j. sbspro.2014.09.278

Ato, M., López, J. J., y Benavente, A. (2013). Un sistema de clasificación de los diseños de investigación en psicología. Anales de Psicología, 29(3), 1038-1059. https://doi.org/10.6018/ analesps.29.3.178511

Barca, A., Peralbo, M., Porto, A., Marcos, J. L., y Brenlla, J. C. (2008). Metas académicas del alumnado de Educación Secundaria Obligatoria (ESO) y Bachillerato con alto y bajo rendimiento escolar. Revista de Educación, 354, 341-368.

Boros, S., y Curseu, P. L. (2012). To be or not to be ... identified. Explorations of students' (dis) identification in a Romanian university. Psihologia Resurselor Umame, 10(1), 57-69.

Cameron, R. B. y Rideout, C. A. (2020). It's been a challenge finding new ways to learn': first-year students' perceptions of adapting to learning in a university environment. Studies in Higher Education, 1-15. https://doi.org/10.1080/03075079.2020.1783525

Cohen, S., \& Janicki-deverts, D. (2010). Can We Improve Our Physical Health by Altering Our Social Networks? Perspect Psychol Sci., 4(4), 375-378. https://doi.org/10.1111/j.17456924.2009.01141.x.Can

Cox, D. W., Bjornsen, A. L. \& Krieshok, T. S. (2015). Occupational Engagement and Academic Major Satisfaction : Vocational Identity's Mediating Role. The Career Development Quarterly, 64, 169-180. Doi: https://doi.org/10.1002/cdq.12049

Di Battista, S., Pivetti, M. y Berti, C. (2014). Engagement in the university context : exploring the role of a sense of justice and social identification. Soc Psychol Educ, 17, 471-490. https://doi. org/10.1007/s11218-014-9255-9

Díaz-mujica, A., García, D., López, Y., Maluenda, J., Hernández, H. y Pérez-Villalobos, M. (2018). Mediación del ajuste académico entre variables cognitivo-motivacionales y la intención de abandono en primer año de universidad [Ponencia]. VIII CLABES Octavo Congreso latinoamericano sobre el abandono en la educación superior. Panamá city, Panamá.

Faleel, S., Tam, C., Lee, T., Har, W. \& Foo, Y. (2012). Stress , Perceived Social Support , Coping Capability and Depression: A Study of Local and Foreign Students in the Malaysian Context. International Journal of Psychological and Behavioral Sciences, 6(1), 1-7.

Figueroa Gazo, P. y Torrado Fonseca, M. (2013). El contexto académico como factor diferenciador en la transición a la universidad. Revista Contrapontos, 13(1), 33-41.

Finn, J. (1993). School engagement y students at risk. State University of New York at Buffalo.

Fredricks, J., Blumenfeld, P. y Paris, A. (2004). School Engagement : Potential of the Concept, State of the Evidence. Review of Educational Research, 74(1), 59-109.

Fredricks, J. y Mccolskey, W. (2012). The Measurement of Student Engagement: A Comparative Analysis of Various Methods and Studen Self-report Instruments. en C. Christenson, S, Reschly, A., Wylie (Ed.), Handbook of Research on Student Engagement (pp. 763-782). New York: Springer-Verlag. https://doi.org/10.1007/978-1-4614-2018-7

Gaeta, M., Cavazos, J., Sánchez, A. y Rosário, P. (2015). Propiedades psicométricas de la versión mexicana del Cuestionario para la Evaluación de Metas Académicas (CEMA). Revista Latinoamericana de Psicología, 47(1), 16-24. https://doi.org/10.1016/S0120-0534(15)30002-9

García, M., González-pienda, J., Núñez, J., González-pumariega, S., Álvarez, L., Roces, C., ... Valle, A. (1998). El cuestionario de metas académicas (C.M.A.). Un instrumento para la evaluación de la orientación motivacional de los alumnos de Educación Secundaria. Revista Aula Abierta,71, 178-202.

Gómez, P., Pérez, C., Parra, P., Oritz, L., Matus, O., McColl, P., ... Meyer, A. (2015). Relación entre el bienestar y el rendimiento académico en alumnos de primer año de medicina. Revista de Médica de Chile, 143(7), 930-937. http://dx.doi.org/10.4067/S0034-98872015000700015 
Goodenow, C. (1993). The Psychological Sense of School Membership Among Adolescents : Scale Development and Educational Correlates. Psychology in the Schools, 30(1), 79-90. https://doi. org/ 10.1002/1520-6807(199301)30:1<79::AID-PITS2310300113>3.0.CO;2-X

Hartley, M. (2011). Examining the Relationships Between Resilience, Mental Health , and Academic Persistence in Undergraduate College Students. Journal of American College Health, 69(7), 596-604. https://doi.org/10.1080/07448481.2010.515632

Herrera, A., Flórez, I., Romero, E. \& Montalvo, A. (2012). Soporte social a cuidadores familiares de personas con enfermedad crónica en. AQUICHAN, 12(3), 286-297.

Hoffman, M., Richmond, J., Morrow, J. y Salomone, K. (2003). Investigating "sense of belonging" in first-year college students. Journal of College Student Retention, 4(3), 227-256.

Itzhaki, Y. (2018). The Different Role of Mentor Support Along the High-School Dropout Process. Youth y Society, 51(7), 981-1008. https://doi.org/10.1177/0044118X18803260

Maluenda, J., Flores-Oyarzo, G., Varas, M. y Díaz, A. (2020). Comportamientos interpersonales del docente asociados al compromiso acadédmico de estudiantes de primer año de ingeniería. Revista de Estudios y Experiencias en Educación Superior, 19(39), 145-161. http://dx.doi. org/10.21703/rexe.20201939maluenda8

Maluenda, J., Florez-Oyarzo, G., Bernardo, A. y Díaz-Mujica, A. (2021). Correlatos conductuales del Compromiso Académico en estudiantes de ingeniería chilenos. Trilogía Ciencia, Tecnología y Sociedad, 13(24). https://doi.org/10.22430/21457778.1754

Maluenda, J., López, Y., Varas, M., Bernardo, A., Díaz, A. \& Moraga, F. (2019). Predictores psicosociales de la intención de abandono en estudiantes de ingeniería chilenos [Ponencia]. IX Congreso Lationamericano sobre Abandono en Educación Superior, Bogotá, Colombia.

Maluenda, J., Moraga, F. y Díaz-Mujica, A. (2019). El rol del estudiante en el fenómeno del Compromiso Académico en Educación Superior. Wimb Lu, 14(1), 81-94. https://doi. org/10.15517/wl.v14i1.35876

Maluenda, J. y Pérez, M. V. (2018). Comportamientos asociados al compromiso académico desde la experiencia de estudiantes de ingeniería [Ponencia]. VIII CLABES Octavo Congreso latinoamericano sobre el abandono en la educación superior, Panamá city, Panamá.

Maluenda, J., Varas, M., Díaz, A. y Bernardo, A. (2020). Propiedades psicométricas del University Student Engagement Inventory en estudiantes de Ingeniería chilenos. Revista Iberoamericana de Diagnóstico y Evaluación - e Avaliação Psicológica, 4(57),77-90. https://doi.org/10.21865/RIDEP57.4.06

Maroco, J., Maroco, A. L., Campos, J. A. D. B. y Fredricks, J. A. (2016). University student's engagement: development of the University Student Engagement Inventory (USEI). Psicologia: Reflexão e Crítica, 29(21). https://doi.org/10.1186/s41155-016-0042-8

Mattanah, J., Ayers, J., \& Brand, B. (2010). A Social Support Intervention to Ease the College. Journal of College Student Development, 51(1), 93-108. https://doi.org/10.1353/csd.0.0116

Musitu, G., Cava, J., \& Valencia, U. De. (2003). El rol del apoyo social en el ajuste de los adolescentes. Intervención Psicosocial, 12(2), 179-192.

Orcasita, L. \& Uribe, A. (2010). La importancia del apoyo social en el bienestar de los adolescentes. Psychologia: Avances de La Disciplina, 4(2), 69-82.

Pérez, M. V., Cobo, R., Matos, L., De Valle, M. y Díaz, A. (2017). Variables cognitivo-motivacionales como predictoras del ajuste a la vida universitaria y de la intención de abandonar estudios en estudiantes de primer año [Ponencia]. VII CLABES Séptimo Congreso latinoamericano sobre el abandono en la educación superior, Córdoba, Argentina.

Salanova, M., Schaufeli, W., Martínez, I. y Bresó, E. (2009). How obstacles and facilitators predict academic performance: The mediating role of study burnout and engagement. Anxiety, Stress \& Coping, 1, 1-18. https://doi.org/10.1080/10615800802609965

Saldaña, M. y Barriga, O. (2010). Adaptación del modelo de deserción universitaria de Tinto a la Universidad Católica de la Santísima Concepción, Chile. Revista de Ciencias Sociales, 16(4), 616-628. 
Schaufeli, W., Martínez, I., Marques, A., Salanova, M. y Bakker, A. (2002). Burnout and Engagemente in university studentes. A Cross-National Study. Journal of Cross-Cultural Psychology, 33(5), 464-481.

Skinner, E., Furrer, C., Marchand, G. y Kindermann, T. (2008). Engagement and Disaffection in the Classroom: Part of a Larger Motivational Dynamic? Journal of Educational Psychology, 100(4), 765-781. https://doi.org/10.1037/a0012840

Tinto, V. (1989). Definir la deserción: Una cuestión de perspectiva. Revista de Educación Superior, $71,35-51$.

Torres, J. y Solberg, S. (2001). Role of Self-Efficacy, Stress, Social Integration, and Family Support in Latino College Student Persistence and Health. Journal of Vocational Behavior, 59, 53-55. https://doi.org/10.1006/jvbe.2000.1785

Tyler, T. R. \& Blader, S. L. (2000). Cooperation Groups: Procedural Justice, Social Identity and Behavioral Engagement. Philadelphia, PA: Psychology Press.

Valle, A., Cabanach, R., Rodríguez, S., Núñez, J., González-pienda, J. A., Solano, P., y Rosário, P. (2009). A motivational perspective on the selfregulated learning in higher education. Global Issues in Higher Education, 20(4), 99-125.

Wang, M. Te, y Eccles, J. S. (2012). Social Support Matters: Longitudinal Effects of Social Support on Three Dimensions of School Engagement From Middle to High School. Child Development, 83(3), 877-895. https://doi.org/10.1111/j.1467-8624.2012.01745.x

Wilkins, S., Mohsin, M., Kratochvil, D. y Stephens, M. (2016). The effects of social identification and organizational identification on student commitment, achievement and satisfaction in higher education. Studies in Higher Education, 41(12), 2232-2252. https://doi.org/10.1080/03075079 .2015 .1034258 